ischaemic attacks and, finally, in 1977, a stroke with a right hemiparesis which disappeared in a month. Three years later, in 1980, nine years after the diagnosis of polycythaemia rubra vera, she had her first depressive episode, with dysphoria, suicidal ideation, psychomotor retardation, persecutory delusions, loss of interest, weight loss, and anergia. No significant life events preceded its onset. Depression ran a chronic fluctuating course, with transient periods of improvement, until she was visited by us, in 1991, still depressed and paranoid. She had $9.9 \mathrm{million} / \mathrm{mm}^{3} \mathrm{RBCs}$, $14000 / \mathrm{mm}^{3}$ WBCs and $900000 / \mathrm{mm}^{3}$ platelets. She had been treated with different antidepressant, antipsychotic and antianxiety drugs. During the last two years polycythaemia had been treated with busulfan (an alkylating agent) and pentoxifylline, which did not change the course of her depression. Previously, she had been treated with aspirin.

Although depression is a well known complication of stroke, the appearance of depression in our case three years later, and its course, point against a causal relationship. Her depression appeared nine years after the diagnosis of polycythaemia and this points against a 'reactive' depression. Clearly, it is possible that the association between polycythaemia and psychotic depression in our case was casual; however, depression seems to be a not uncommon complication of polycythaemia rubra vera, according to the haematology literature cited by Murray \& Hodgson.

Marco Mazzoli

Psychiatry Service

Public Hospital 'Morgagni'

47100 Forli

Italy

via Pozzetto 17

48010 Castiglione de Cervia RA

Italy

\section{Phobic disorders and benzodiazepines in the elderly}

SIR: In his interesting paper Lindesay (Journal, October 1991, 159, 531-541) fails to consider the possibility that benzodiazepine drugs may have been the cause of the late-onset phobias in some of his patients. In 11 of 18 in whom the main fear began after the age of 65 years, it followed a physical event, and in five, some other traumatic exposure such as a mugging, a fall or being trapped in a lift. In all these situations it is not unusual (although of course wrong) for benzodiazepine drugs to be given, and not infrequently they continue to be given subsequently. Nineteen of Dr Lindesay's cases were taking psychotropic medication, most commonly a benzodiazepine, but we do not know from his data what the relationship of taking these drugs was to the onset or aggravation of the symptoms.
The natural history of anxiety symptoms following illnesses or trauma is towards natural resolution and when this does not happen one must always consider what may be keeping them going. In some patients, perhaps because of their individual vulnerability, the cause is benzodiazepine drugs and if this is the case these patients can be totally relieved of their symptoms. This problem arises at all ages and is illustrated by the following example in an elderly patient.

Case report. A lady of 75 had always been rather phobic of lifts and of trains but for two years she had suffered from severe anxiety, panic, terror and misery. This followed an operation on her knee, since when she had taken lorazepam $2 \mathrm{mg}$ daily. She was so frightened that when travelling in the car with her husband she felt she wanted to jump out of the car if he stopped at traffic lights, and a measure of the intensity of this feeling was that her husband fixed a special lock on the door. She could not be left alone in the car while he went into a shop to buy a newspaper. Her tablets were stopped over a period of four weeks with suitable explanation of what to expect by way of withdrawal symptoms, and at the end of five weeks she said it had been "the worst five weeks of my life". At eight weeks there had been a few spells of feeling normal and at 12 weeks she had been in a lift although severe insomnia persisted. At 16 weeks she was completely well, happy and free of symptoms. She could sit in the car long enough for her husband to have his hair cut.

This is not a new idea. Ashton (1984) pointed out that many patients develop phobias for the first time after a period of regular benzodiazepine use and that these symptoms disappear when the drug is withdrawn. Her paper gives references to a similar view expressed previously by Lader, Tyrer and others. The above example is one among many such patients, of all ages, and although it is not always easy to wean patients from their tablets it can usually be done with appropriate support and explanation and these patients become some of the most grateful a psychiatrist can have.

AsHTon, H. (1984) Benzodiazepine withdrawal: an unfinished story. British Medical Journal. 288, 1135-1140.

The London Hospital Medical College

SAmuel I. COHEN Turner Street

London EI $2 A D$

\section{NMS complicated by diazepam}

SIR: Neuroleptic agents are used extensively in psychogeriatric practice, including the management of behavioural problems in Alzheimer's disease. Serby (1986) reported NMS in a patient with Alzheimer's disease. Finucane et al (1984) reported NMS in an 81-year-old man, induced by haloperidol. This communication pertains to a 67-year-old gentleman who 
developed NMS and further complications due to the usage of diazepam in the early stages of NMS.

Case report. This 67-year-old man was admitted to a general hospital for management of NMS. He carried a diagnosis of chronic schizophrenia as well as glaucoma and non-insulindependent diabetes.

The patient had originally presented to a local general hospital with agitation and rambling speech, and the emergency services transferred him to the catchment area psychiatric hospital where he received two doses of haloperidol $2.5 \mathrm{mg}$ i.m. for aggressive behaviour in addition to alprazolam $0.25 \mathrm{mg}$ t.i.d. and trifluoperazine $10 \mathrm{mg}$ p.o. q.h.s. Because of an increasing CPK (3205 IU/C), fever $\left(38^{\circ} \mathrm{C}\right)$ and confusion within 48 hours, he was transferred to the local general hospital due to suspicion of NMS. The patient was investigated for possible cardiac cause of his elevated CPK and the tests were negative. The patient was then placed on diazepam $10 \mathrm{mg}$ i.m. q.4.h. p.r.n. and chlordiazepoxide $25 \mathrm{mg}$ p.o. i.m. q.4.h. p.r.n. for control of agitation. He received diazepam 5-10 mg i.m. on six occasions within a time frame of 24-72 hours as well as chlordiazepoxide $25 \mathrm{mg}$ i.m. on one occasion. The patient received this for very agitated behaviour, trying to remove shackles, restlessness, yelling, and at times expressing paranoid ideas. He would respond by getting drowsy, sleeping for long periods and waking with further agitation. He gradually became comatose and was transferred to a teaching hospital for management of suspected NMS.

On arrival, the patient had a blood pressure of $100 / 10$, respiratory rate of 30 and heart rate of 100 . Examination of the head and neck revealed neck stiffness and increased tone in the extremities. Pupils were slightly reactive and fundi were difficult to assess. Gag was intact. Examination of the chest, cardiovascular systems and abdomen revealed no abnormalities. Spontaneous movements of both arms were present.

The patient underwent comprehensive tests including CT of the head and lumbar puncture which were both negative. A drug screen was positive for benzodiazepines, and EEG findings were consistent with diazepam-induced encephalopathy. The patient was managed conservatively with emphasis on hydration. The patient's level of consciousness gradually improved over a period of ten days; he was then referred to the psychogeriatric service for follow-up care.

Therapy in NMS is largely supportive. This consists of stopping the offending agent, correcting fluid and electrolyte imbalances, monitoring vital signs, fluid intake and urinary output, and reducing the core body temperature.

The use of benzodiazepine in NMS has been advocated based on their efficacy in a small number of patients (Fricchione et al, 1983; Lew et al, 1983). However, it should be remembered that they bind tightly to plasma proteins and are highly lipophilic. This means that they are hard to remove from the body, especially in the elderly. Benzodiazepines like oxazepam and lorazepam have no active metabolites, and the elimination half lives of these compounds is in the short to intermediate range (5-20 hours), but for diazepam it is $20-100$ hours. The metabolism of diazepam is therefore more affected by the presence of liver disease, the extremes of age, or drug interactions. If benzodiazepines are used in the management of NMS, the longer half-life drugs, e.g. diazepam, should probably be given once a day only. Shorter half life drugs, e.g. lorazepam, oxazepam, could probably be given in divided doses two to three times a day. These dosage regimens are even more important while treating the elderly.

Finucane, P. \& MURPHy, S. F. (1984) Neuroleptic malignant syndrome-common or rare? (Abstract). Irish Journal of Medical Science, 153, 156.

Fricchione, G. L., Cassem, N. H., Hooberman, D. et al (1983) Intravenous lorazepam in neuroleptic-induced catatonia. Journal of Clinical Psychopharmacology, 3, 338-342.

LEW, T. Y. \& TOLLEFSON (1983) Chlorpromazine-induced neuroleptic malignant syndrome and its response to diazepam. Biological Psychiatry, 18, 1441-1446.

SERBY, M. Neuroleptic malignant syndrome in Alzheimer's disease. Journal of the American Geriatric Society. 34, 895-896.

University of Western Ontario

V. R. VelAMOOR

375 South Street

London

Ontario

Canada

\section{CORRIGENDUM}

Journal, November 1991, 159,645. “A. C. Almatura”, listed as additionally involved in the preparation of the paper, should read "A. C. Altamura".

\section{A HUNDRED YEARS AGO}

\section{"The Hypnotised Lobster"}

ThE following appeared in Punch, December 19th, 1891:

[Mr. Ernest Hart said, in a recent lecture, that snakes, frogs, and lobsters could be hypnotised like human beings.]
'Tis the voice of the lobster I hear him complain, That hypnotic suggestion is on me again;

I was mesmerised once, and behold, since that time,

I have yielded myself to suggestions of crime;

I have compassed the death of an innocent "dab," And attempted to poison an elderly crab. 